
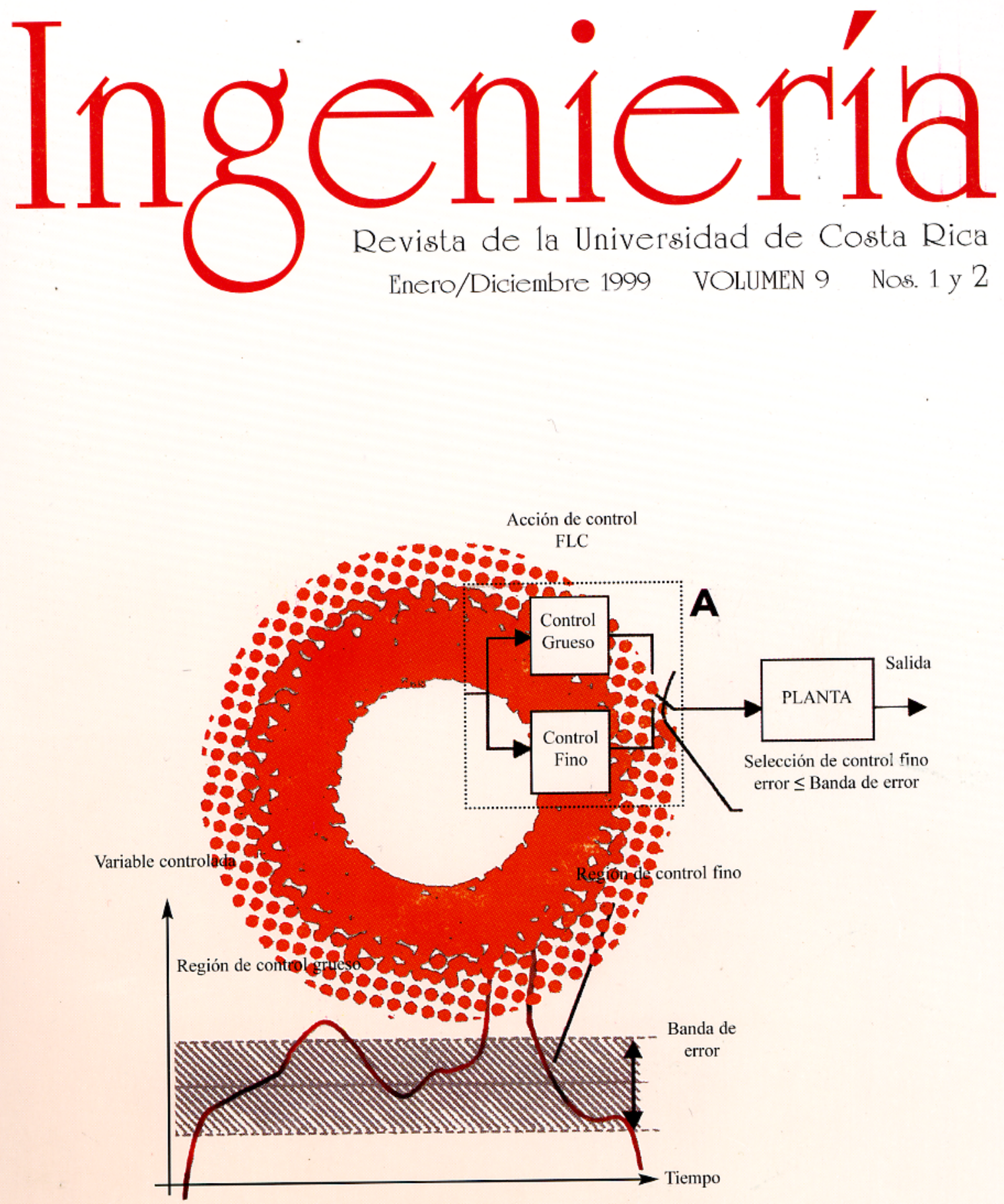


\section{INGENIERIA}

Revista Semestral de la Universidad de Costa Rica

Volumen 9, Enero/Diciembre 1999 Números 1 y 2

\section{DIRECTOR}

Rodolfo Herrera J.

CONSEJO EDITORAL

Víctor Hugo Chacón P.

Ismael Mazón $\mathrm{G}$.

Domingo Riggioni C.

\section{CORRESPONDENCIA Y SUSCRIPCIONES}

Editorial de la Universidad de Costa Rica

Apartado Postal 75

2060 Ciudad Universitaria Rodrigo Facio

San José, Costa Rica

\section{CANJES}

Universidad de Costa Rica

Sistema de Bibliotecas, Documentación e Información

Unidad de Selección y Aquisiciones-CANJE

Ciudad Universitaria Rodrigo Facio

San José, Costa Rica

\section{Suscripción anual:}

Costa Rica: $₫ 1000,00$

Otros países: US $\$ 30,00$

Número suelto:

Costa Rica: $\not 750,00$

Otros países: $\$ 20,00$

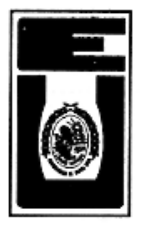




\title{
SIMULACIÓN DE UN SISTEMA DE TRANSMISIÓN DE DATOS
}

\author{
Arturo Ramirez $P$ ?
}

\section{Resumen}

En este trabajo se presenta una simulación por "software" de un sistema digital de transmisión de datos utilizando la herramienta llamada LabVIEW ${ }^{\star}$ para Windows ${ }^{\oplus}$, versión 5.0, de National Instruments. El sistema modela un generador binario de pulsos (o bits), un filtro de transmisión, un filtro de canal y un filtro receptor. Se escogieron y compararon los desempeños de filtros de Butterworth y Chebyshev de distintos órdenes, los cuales vienen ya incluidos con la herramienta. Se agrega un componente de ruido blanco gaussiano para simular las condiciones reales de transmisión. El trabajo se enfoca en el cálculo de las probabilidades de error que se presentan cuando un bit recibido es decodificado erróneamente por un convertidor Analógico/Digital, el cual también se modela en este trabajo. Los resultados indican que las probabilidades de error dependen fuertemente de la razón señal a ruido, aunque son ligeramente sensibles al orden del filtro. Se encontró una mayor probabilidad de error para los filtros de Chebyshev en relación con los de Butterworth para bajos órdenes, lo que parece atribuible a la interferencia entre simbolos.

\section{Summary}

In this work, a digital transmission data system is software simulated by the use of National Instruments' LabVIEW 5.0, Windows version. The systems models a binary pulse generator, a transmission filter, a channel filter and a receiver filter. Butterworth and Chebyshev filters were chosen to compare their performances. A gaussian white noise component is added to the model in order to simulate the actual transmission conditions. This work focuses on the calculation of error probabilities that arise when a received bit is decoded erroneously by the Analogic-to-Digital converter, which is also modeled in this work. The results show that the error probabilities strongly depend on the signal-to-noise ratio, although they seem to be lightly sensitive to the filter order. A higher error probability was found for the Chebyshev filters against the Butterworth ones for low orders. The interference between symbols may
be the cause for this.

\section{INTRODUCCIÓN}

La rápida proliferación de redes de computadoras ha traído consigo la necesidad de mejorar los sistemas de transmisión de datos para minimizar las pérdidas de información y los tiempos de retransmisión. Esto es particularmente urgente en sistemas que trabajan con datos altamente sensibles, como lo son los bancos y las agencias gubernamentales estratégicas. Por este motivo, el diseño de los sistemas de transmisión se ha ido sofisticando a pasos agigantados en los últimos años. Es común ahora encontrar redes que manejan datos por fibra óptica a muy altas velocidades y con baja taza de errores. No obstante, este tipo de sistemas es aún relativamente caro $\mathrm{y}$ no necesariamente accesible a todos los sectores de producción o de servicios. Por tal motivo, la tecnología de transmisión basada en cobre es todavía ampliamente utilizada debido a su costo bajo, aunque hay que pagar el precio de su mayor taza de errores de transmisión debido principalmente al ruido que se genera en las líneas de transmisión. Este trabajo pretende analizar el efecto del ruido, supuesto como ruido blanco gaussiano, en el cálculo de la probabilidad de error para un sistema digital binario.

\section{CONSIDERACIONES TEÓRICAS}

A continuación se presenta el análisis y el diseño de una técnica de modulación de pulsos 
discretos que se puede usar para transmitir la salida de una fuente discreta sobre un canal de

banda base. El esquema de modulación que se tratará aquí es el de amplitud de pulso (PAM), el

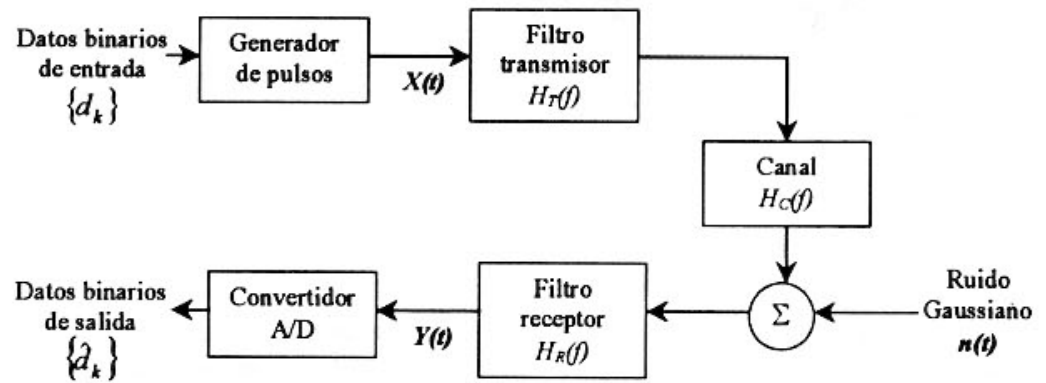

Figura No. 1: Sistema de transmisión de datos binarios en banda base.

cual es muy eficiente en cuanto a utilización de potencia y ancho de banda (Ref. No. 1). La figura No. 1 muestra los elementos principales de un sistema PAM binario en banda base.

La entrada al sistema es una secuencia de datos binarios con velocidad de transmisión de bits $r_{b}$ y duración de bit $T_{b}$. La salida del generador de pulsos es la siguiente función:

$$
X(t)=\sum_{k=-\infty}^{\infty} a_{k} p_{g}\left[t-k T_{b}\right]
$$

en donde $p_{g}(t)$ es un pulso básico cuya amplitud $a_{k}$ es igual a $a$ si el $k$-ésimo bit de entrada es un 1 , 0 - $a$ si el $k$-ésimo bit de entrada es un 0 . Nótese que se está usando un sistema bipolar para los pulsos. Se supone que $p_{g}(t)$ esta normalizado de modo que $p_{g}(0)=1$.

La señal $X(t)$ pasa a través del filtro transmisor $H_{T}(f)$ y luego sobre el canal que añade ruido aleatorio. La señal ruidosa llega al filtro receptor $H_{R}(0)$ y la salida $Y(t)$ es muestreada por el convertidor analógico/digital (A/D). La secuencia de bits transmitidos es regenerada por el convertidor A/D tomando como base los valores de $Y(t)$ cuya función se puede escribir de la forma:
$Y(t)=\sum_{k=-\infty}^{\infty} A_{k} p_{r}\left[t-t_{d}-k T_{b}\right]+n_{0}(t)$

donde:

- $A_{k}=K_{c} a_{k}, K_{c} p_{r}\left(t-t_{d}\right)$ es la respuesta del sistema cuando la entrada es $p_{g}(t), t_{d}$ es un tiempo arbitrario de retraso y $n_{0}(t)$ es el ruido en la entrada del receptor. $K_{c}$ es una constante de normalización que hace $p_{r}(0)=1$.

El convertidor muestrea $Y(t)$ en $t_{m}=m T_{b}+t_{d}$ y el $m$-ésimo bit se genera comparando $Y\left(t_{m}\right)$ con el umbral (que es 0 para sistemas de transmisión de datos binarios). La entrada al convertidor en el instante $t_{m}$ es:

$Y\left(t_{m}\right)=A_{m}+\sum_{k \neq m} A_{k} p_{r}\left[(m-k) T_{b}\right]+n_{0}\left(t_{m}\right)$

Aquí, $A_{m}$ representa el $m$-ésimo bit transmitido mientras que la sumatoria representa el efecto residual de los otros bits transmitidos sobre el $m$ ésimo bit. Este efecto es llamado interferencia entre simbolos (ISI) y constituye un efecto de acoplamiento entre los voltajes de los pulsos. El último término representa el ruido.

En ausencia de ruido y de ISI, el $m$-ésimo bit transmitido puede ser decodificado correctamente con base en $Y\left(t_{m}\right)$ puesto que $Y\left(t_{m}\right)=K_{c} a_{m} \mathrm{y} \quad a_{m} \quad$ está relacionado 
únicamente con el m-ésimo bit de entrada. El ruido y el ISI introducen errores en la salida. Los objetivos principales en el diseño de sistemas PAM son seleccionar los filtros transmisores y receptores de modo que se minimicen estos efectos. No obstante, en este trabajo la atención se concentrará mayormente en el efecto del ruido sobre el sistema puesto que es quizá el efecto más importante, aunque la acción del ISI se puede hallar al comparar los desempeños de los dos tipos de filtros utilizados, según se verá más adelante. Se supone que son conocidas las caracteristicas fisicas del canal y las características estadísticas del ruido. Entonces, se habrá de escoger las formas $p_{g}(t)$ y $p_{r}(t)$ de los pulsos y las funciones de transferencia $H_{T}(f)$ y $H_{R}(0)$ de los filtros de modo que se optimice el desempeño del sistema, dejando la probabilidad de error debajo de un cierto valor especificado.

La meta ahora es obtener una expresión para la probabilidad de error en un bit. La probabilidad de error, que se usa como una medida del desempeño de sistemas PAM binarios, se define como:

$$
P_{e}=P\left[\hat{d}_{k} \neq d_{k}\right]
$$

Nótese que en el $m$-ésimo tiempo de muestreo, la entrada al convertidor A/D es, en ausencia de ISI:

$$
Y\left(t_{m}\right)=A_{m}+n_{0}\left(t_{m}\right), \quad t_{m}=m T_{b}+t_{d}
$$

La salida del convertidor será 1 o 0 dependiendo de si $Y\left(t_{m}\right)>0 \quad$ o $\quad Y\left(t_{m}\right)<0$, respectivamente. Definiendo $d_{m}$ como el $m$ ésimo bit de entrada, la probabilidad para decodificar incorrectamente este bit en el receptor está dada por:

$$
\begin{aligned}
P_{e}= & P\left[Y\left(t_{m}\right)>0 \mid d_{m}=0\right] P\left(d_{m}=0\right)+ \\
& P\left[Y\left(t_{m}\right)<0 \mid d_{m}=1\right] P\left(d_{m}=1\right)
\end{aligned}
$$

Si la ocurrencia de bits es equiprobable y con $Y\left(t_{m}\right)=A+n_{0}\left(t_{m}\right)$ cuando $d_{m}=1 \mathrm{y}$ $Y\left(t_{m}\right)=-A+n_{0}\left(t_{m}\right)$ cuando $d_{m}=0$, con $A=K_{c} a$, se tiene:

$$
P_{.}=\frac{1}{2} P\left[n_{0}\left(t_{m}\right)<-A\right]+\frac{1}{2} P\left[n_{0}\left(t_{m}\right)>A\right]
$$

Esto es:

$$
P_{\odot}=\frac{1}{2} P\left[\left|n_{0}\left(t_{m}\right)\right|>A\right]
$$

Se supone arbitrariamente que el ruido es Gaussiano con media cero y varianza $N_{0}$. Entonces, la probabilidad de error se escribe:

$$
\begin{aligned}
P_{.} & =\frac{1}{2} \int_{|x|>A}^{\infty} \frac{1}{\sqrt{2 \pi N_{0}}} \exp \left(-x^{2} / 2 N_{0}\right) d x \\
& =\int_{A}^{\infty} \frac{1}{\sqrt{2 \pi N_{0}}} \exp \left(-x^{2} / 2 N_{0}\right) d x
\end{aligned}
$$

Con el cambio de variable $z=x / \sqrt{N_{0}}$ se obtiene la expresión:

$$
P_{e}=\int_{A / \sqrt{N_{0}}}^{\infty} \frac{1}{\sqrt{2 \pi}} \exp \left(-z^{2} / 2\right) d z=Q\left(\frac{A}{\sqrt{N_{0}}}\right)
$$

donde $Q(u)$ es la función complementaria de error:

$$
Q(u)=\int_{u}^{\infty} \frac{1}{\sqrt{2 \pi}} \exp \left(-z^{2} / 2\right) d z
$$

Se observa cntonces que para minimizar la probabilidad de error, se debe maximizar $A / \sqrt{N_{0}}$, que no representa otra cosa que la razón señal a ruido. Además, es posible probar (Ref. No. 1) que para cl ruido Gaussiano blanco, la probabilidad de error se minimiza cuando los filtros transmisor y receptor tienen 
características de frecuencia iguales, aunque pueden diferir en cuanto a ganancia. En la simulación se utilizarán idénticos filtros para el transmisor, el receptor y el canal. del funcionamiento del sistema. La figura No. 2 muestra el aspecto de la interfaz en donde se destacan los controles de las señales y los indicadores del tipo encontrado en los osciloscopios.

Los controles llamados Altura, Ancho y Retraso

\section{Sistema de Transmisión v. 3.0 por Arturo Ramirez Porras, acıma, Universidad de Costa Fíca. Jun99}

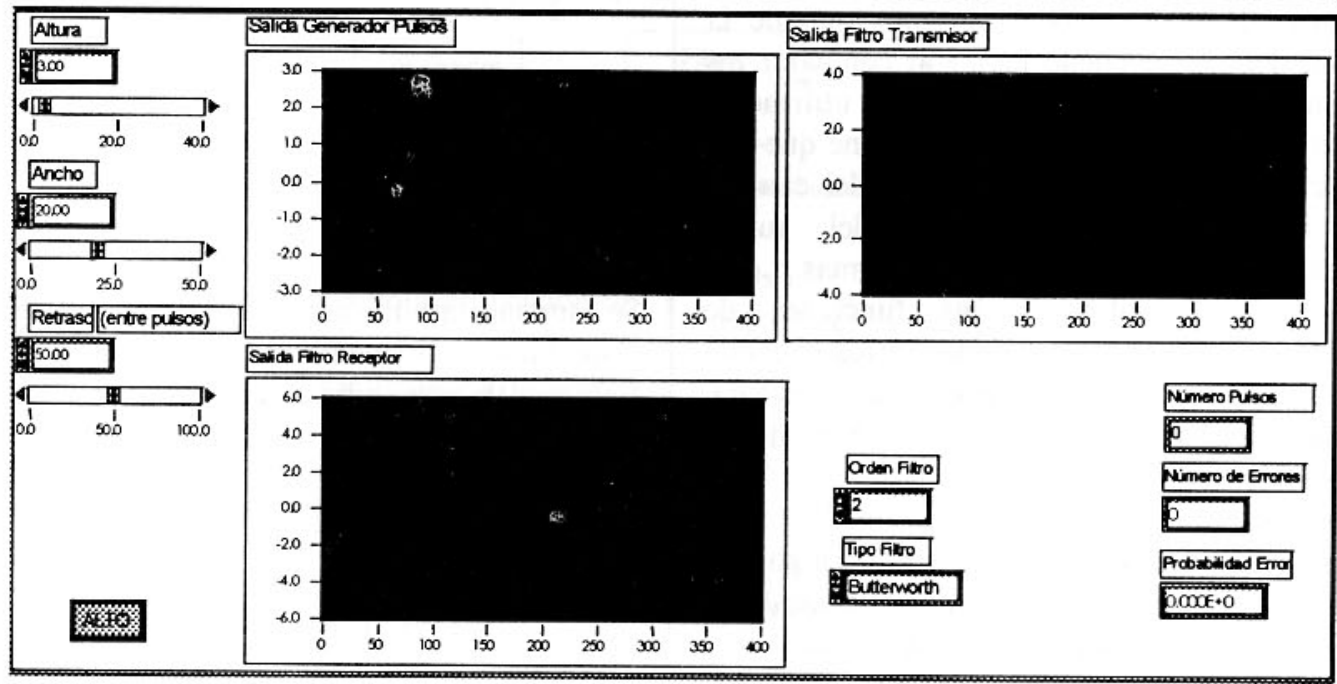

Figura No. 2: Interfaz gráfica del simulador

\section{DESCRIPCIÓN DEL SIMULADOR}

Con base en la teoría anteriormente citada, se procedió a desarrollar un simulador cuyas metas principales eran las siguientes:

1. Probar que si la razón señal a ruido aumentaba, la probabilidad de error disminuía.

2. Comparar el desempeño de dos tipos de filtros. Se escogieron arbitrariamente los de Butterworth y los de Chebyshev.

El programa utilizado es el LabVIEW de National Instruments para Windows que tiene entre otras ventajas el que se pueden programar interfaces gráficas de instrumentos virtuales con suma facilidad. La programación de los instrumentos se hace gráficamente (en lenguaje llamado $G$ ) de forma similar a los diagramas de bloques. Esto hace que sea fácil la comprensión controlan el alto, ancho y separación de los bits. Orden Filtro representa el orden del filtro digital "pasobajo" asociado con el transmisor, el canal y el receptor. Tipo Filtro controla el tipo del filtro. Se escogieron los filtros de Butterworth y Chebyshev, cuyas funciones de transferencia normalizadas son las siguientes:

$$
F_{B u t}(\omega)=\frac{1}{\sqrt{1+\left(\frac{\omega}{\omega_{0}}\right)^{n}}}, \quad F_{\text {Chev }}(\omega)=\frac{1}{\sqrt{1+C_{n}^{2}\left(\omega / \omega_{0}\right)}}
$$

Aquí, $n$ es el orden del filtro, $\omega_{0}$ es la frecuencia de corte y $C_{n}(x)$ es el polinomio de Chebyshev de orden $n$ cuya expresión matemática puede encontrarse en los libros de fórmulas matemáticas estándares (como la Ref. No. 2). En la figura No. 3 se presentan las dos funciones para algunos órdenes. 


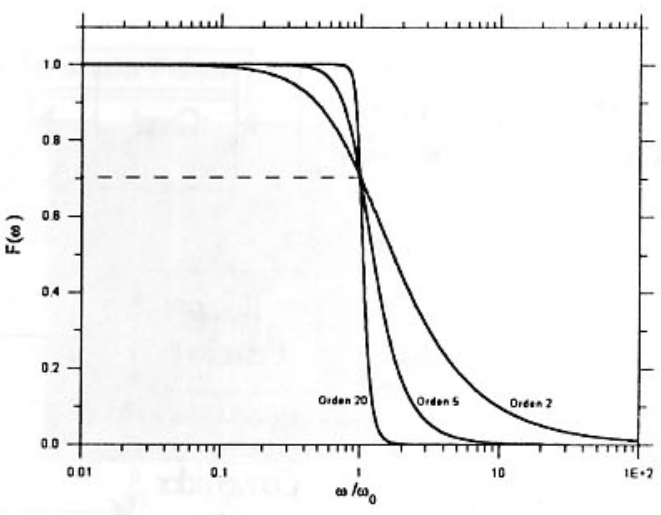

(a)

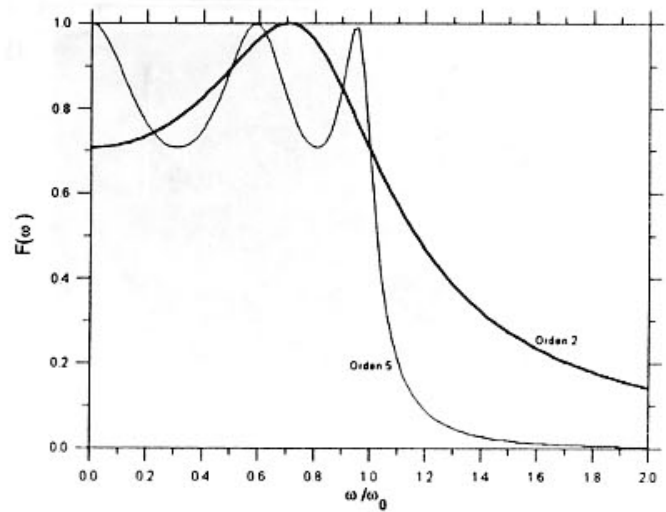

(b)

Figura No. 3: Funciones de transferencia normalizadas para los filtros de (a) Butterworth y (b) Chebyshev.

La ventaja de los filtros de Butterworth es que existe estabilidad en la respuesta a las frecuencias de paso, aunque la transición hacia las frecuencias de corte es más bien lenta. Con los filtros de Chebyshev existe una mejor transición que asemeja más al filtro paso bajo ideal, aunque se presenta el fenómeno de rizado en bajas frecuencias.

En la interfaz gráfica se muestran los oscilogramas de las señales de salida del generador de pulsos, de salida del filtro transmisor de datos y del filtro receptor. En este último, se incluyen los efectos del ruido, según se mostró en la figura No. 1.
La figura No. 4 presenta el diagrama de bloques básico para la construcción del simulador. Los parámetros de entrada son el alto y la duración del bit, el tiempo entre bits y el orden y tipo de los filtros. Con estos datos se genera una misma secuencia arbitraria de pulsos que pasa por el filtro transmisor y luego por el canal. Se agrega el ruido gaussiano justo antes de entrar al filtro receptor. La salida ingresa al convertidor A/D y se compara el tren obtenido con el tren de pulsos de inicio. Si los patrones no coinciden, se cuentan los bits erróneos. La probabilidad de error se calcula repitiendo el proceso un cierto número de veces (entre cincuenta mil y ciento treinta mil bits generados) y acumulando el total de bits erróneos. Este resultado se divide finalmente entre el número de bits transmitidos. 


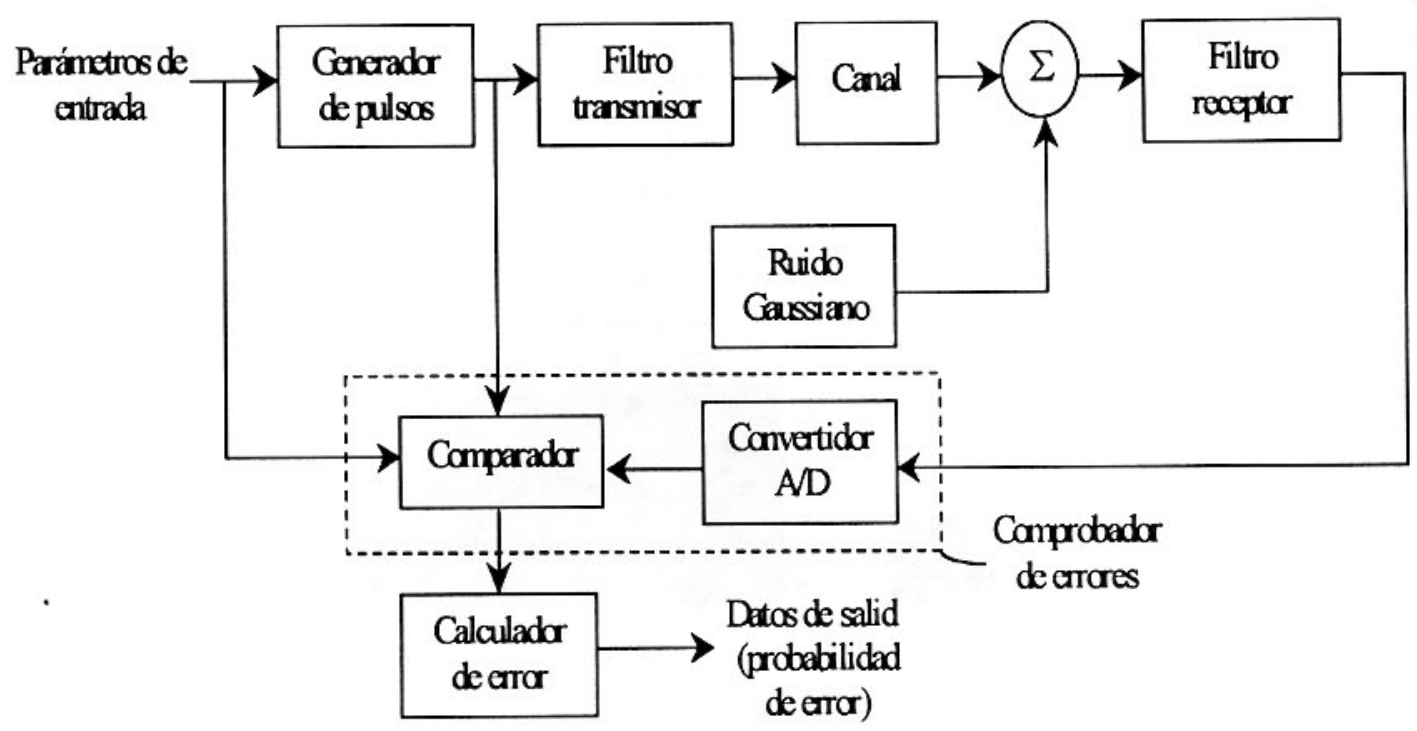

Figura No. 4: Diagrama de bloques del simulador.

\section{RESULTADOS}

La figura No. 5 muestra las señales típicas obtenidas para el caso de un tren de pulsos binario de 3 unidades de altura (mostrado en (a)), 20 unidades de tiempo (UT, por ejemplo, $\mu \mathrm{s}$ ) de ancho y 50 UT de separación.

Todos los oscilogramas representan, por ejemplo, el voltaje en función del tiempo. En (b) y (d) se muestran las señales que se obtienen luego del transmisor y receptor, respectivamente. Éstos se modelaron como filtros de segundo orden de Butterworth, al igual que el canal transmisor (cuya señal no se muestra por ser similar a la del transmisor). La frecuencia de muestreo $f_{s}$ para los filtros (ya sea de Butterworth o Chebyshev) en todos los casos analizados fue arbitrariamente igual a $1 \mathrm{UT}^{-1}$, es decir, mayor que la frecuencia de los pulsos. La frecuencia de corte $f_{c}$ se igualó a $0,125 \mathrm{UT}^{-1}$ en todos los casos, lo cual siempre observaba el criterio de Nyquist $\left(0 \leq f_{c} \leq(1 / 2) f_{s}\right)$. En (c) se muestra la señal proveniente del ruido gaussiano puro con media cero y variancia $N_{0}$ igual a la unidad. En todas las simulaciones se mantuvo el valor indicado de varianza, por lo que las razones señal a ruido $\left(A / \sqrt{N_{0}}\right)$ mencionadas previamente son iguales al alto de los pulsos. Es decir, una señal de 3 unidades de altura tiene una razón señal a ruido de 3 . 


\section{Salida Generador Pulsos}

(a)

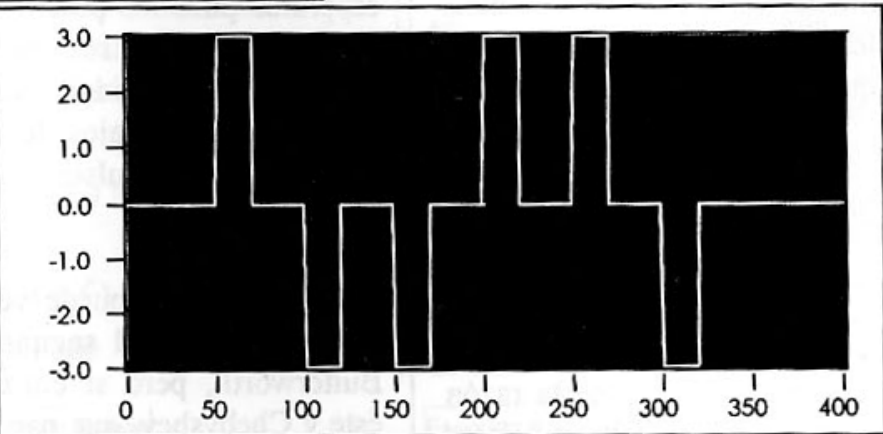

Salida Filtro Transmisor (Butterworth, $2^{\circ}$ orden)

(b)

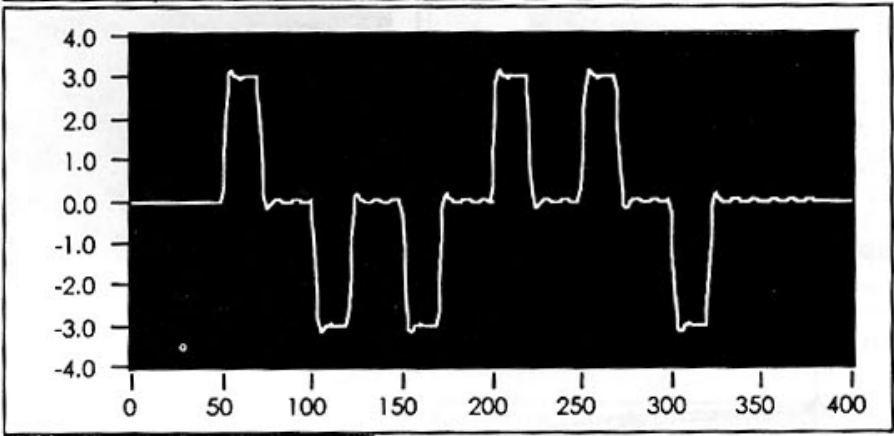

Ruido Gaussiano, Sigma $=1$

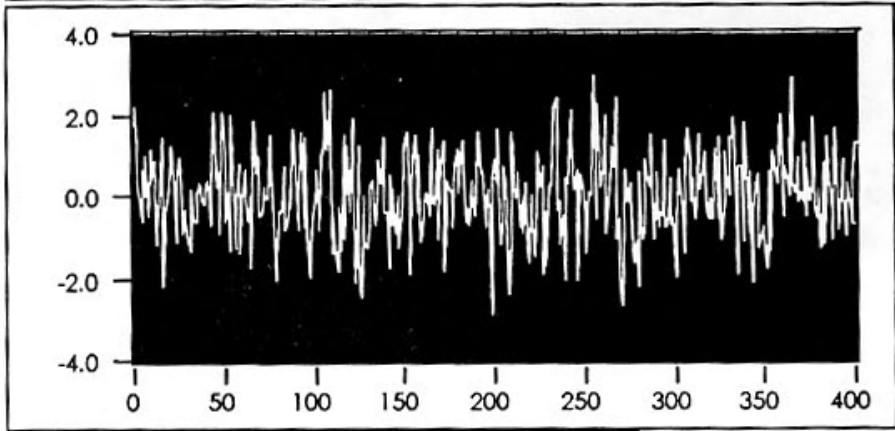

Salida Filtro Receptor (Butterworth, $2^{\circ}$ orden)

(c)

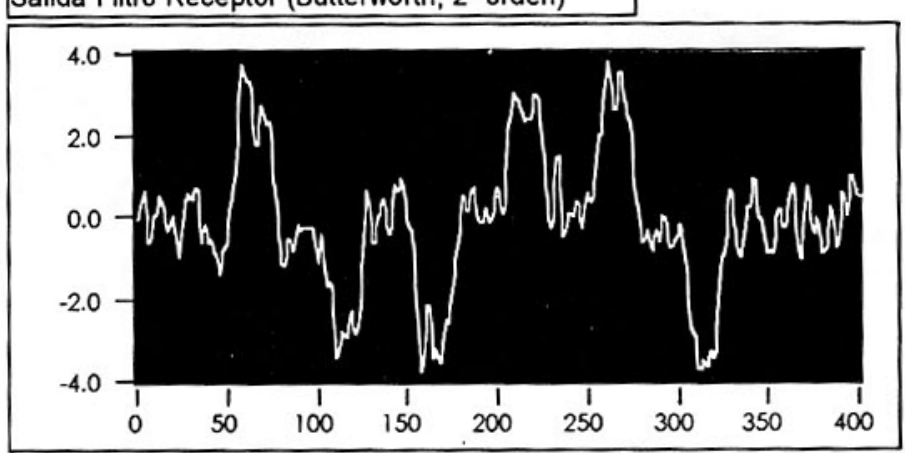

Figura No. 5: Oscilogramas típicos obtenidos por el simulador. (a) Tren de pulsos; (b) Señai a la salida del filtro transmisot tipo Butterworth de segundo orden; (c) Señal del ruido gaussiano de variancia unitaria; (d) Señal de salida a la salida del filtrc receptor del mismo tipo que el transmisor. 
Sin embargo, para los resultados que se presentarán a continuación, se calcularán dichas razones en decibeles $(\mathrm{dB})$ calculados a partir de voltajes, de modo que:

$$
A_{d B}=20 \log \left(\frac{A}{\sqrt{N_{0}}}\right)
$$

La figura No. 6 muestra los datos obtenidos para la probabilidad de error en función de la razón señal a ruido para varios casos de filtros. Nótese que los datos van desde $-20 \mathrm{~dB}$ (es decir, razón de 0,1 ) hasta aproximadamente $+7,6 \mathrm{~dB}$ (razón de 2,4 ). El número máximo típico de pulsos generados era del orden de cien mil, por lo que el error mínimo detectable era de $10^{-5}$.

Se puede notar que para pulsos menores que el ruido, la probabilidad tiende a 0.5 , como es de esperarse pues los pulsos quedan ocultados por el ruido. Para pulsos bastante mayores que el ruido, la probabilidad cae exponencialmente a valores bastante bajos, lo que indica una buena detección de los pulsos, como se puede observar en la figura No. 5.

En la gráfica se puede ver que no existe gran diferencia entre el segundo y quinto orden de Butterworth, pero sí entre mismos órdenes de éste y Chebyshev, que parece dar probabilidades de error mayores. Según Shanmugam (Ref. No. 1), esto se debe a que si bien los filtros de Butterworth ocupan mayor ancho de banda que los de Chebyshev, esto se compensa con que entonces los pulsos decaen más rápidamente en dominio del tiempo y por tanto causan menor cantidad de interferencia entre símbolos.

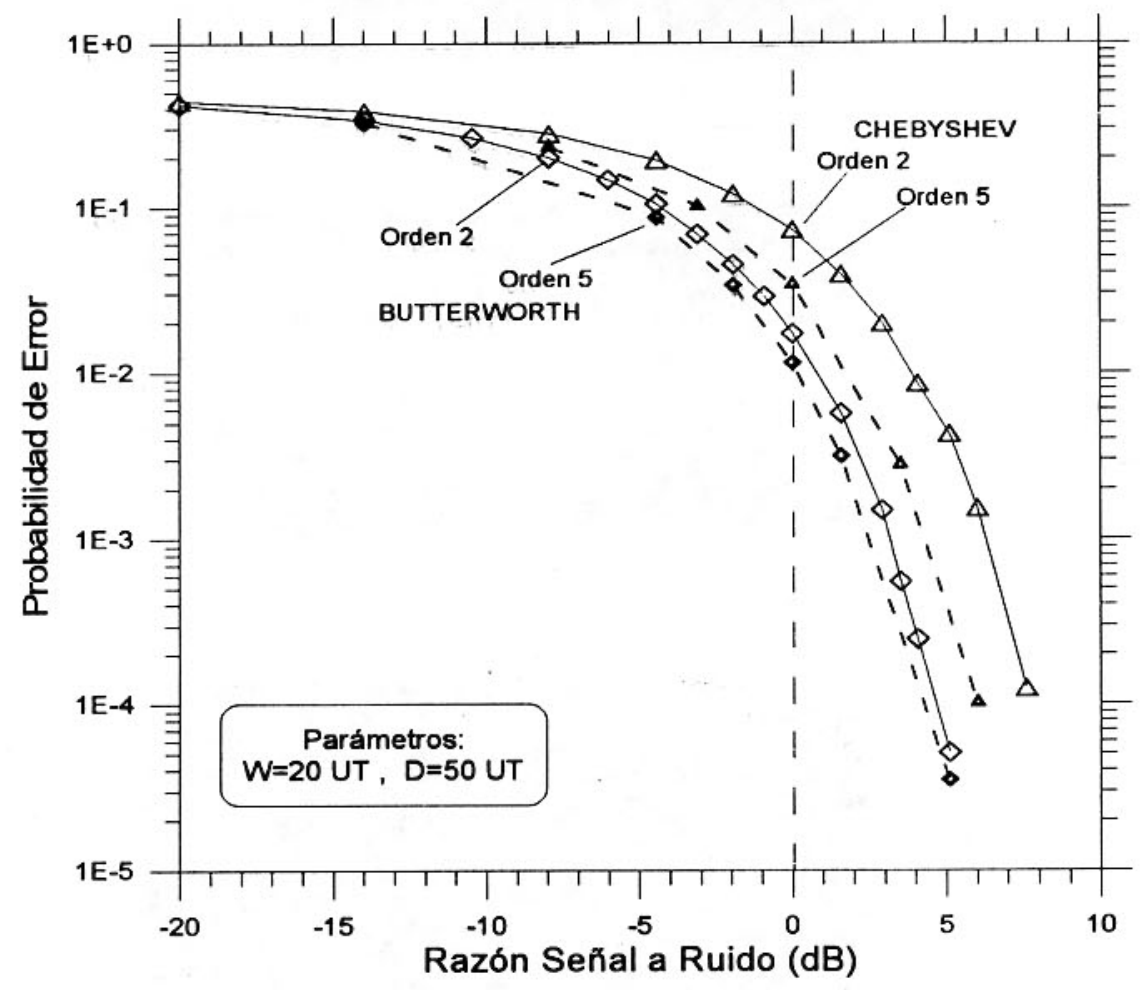

Figura No. 6: Resultados obtenidos para la probabilidad de error como función de la razón señal a ruido para distintos casos de filtros y órdenes, según están indicados en el gráfico. 
A continuación se presentan algunos espectros de potencia obtenidos con el instrumento que para tal efecto viene incluido en el LabVIEW. Este instrumento calcula la función:

$$
S_{X X}(f)=X^{*}(f) \times X(f)=|X(f)|^{2}
$$

donde:

$f$ es la frecuencia, $X(f)=F\{x(t)\}$ es la transformada de Fourier de una señal $x(t)$ y

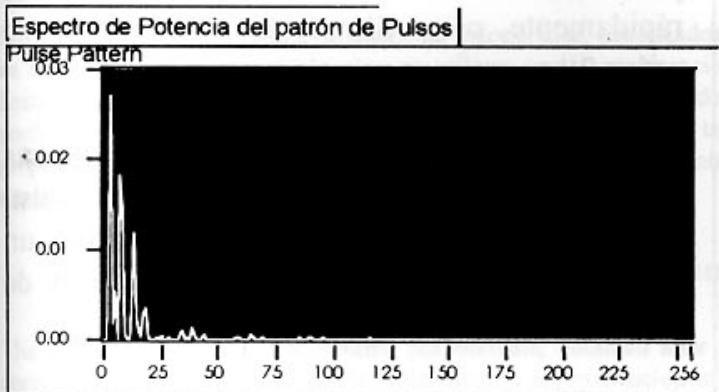

(a)

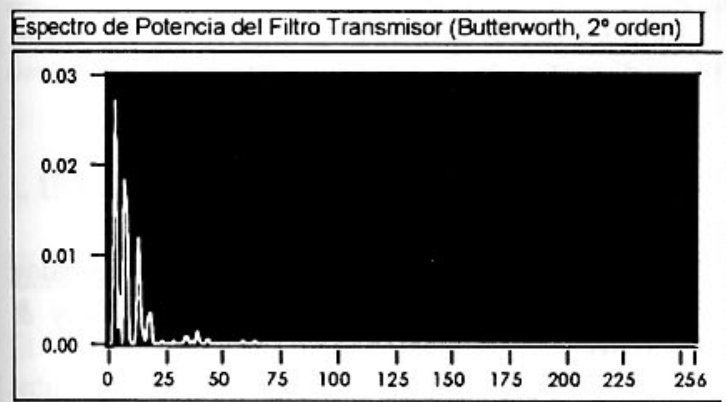

(c)

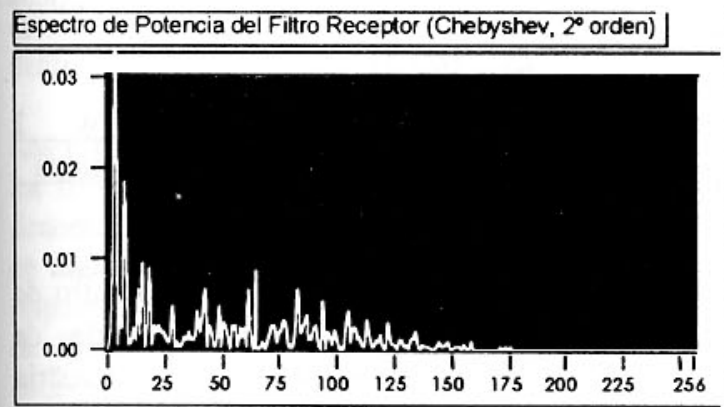

(e)
$X^{*}(f)$ es su complejo conjugado. Puesto que se supone que las señales en el tiempo representan voltajes, el instrumento está diseñado (Ref. No. 3) para dar los resultados en Energía/(Tiempo-Ohm). Si se considera que la unidad de tiempo aquí utilizada es UT que puede representar, por ejemplo, $\mu \mathrm{s}$, entonces los datos estarán dados en unidades de Watts/Ohms. En realidad el instrumento realiza sus cálculos con la ayuda de la transformada rápida de

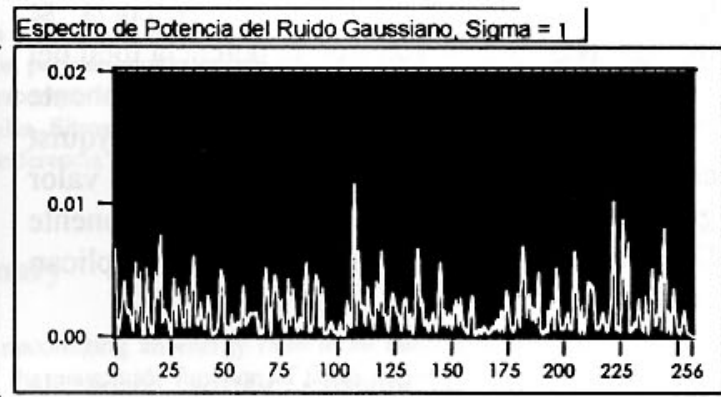

(b)

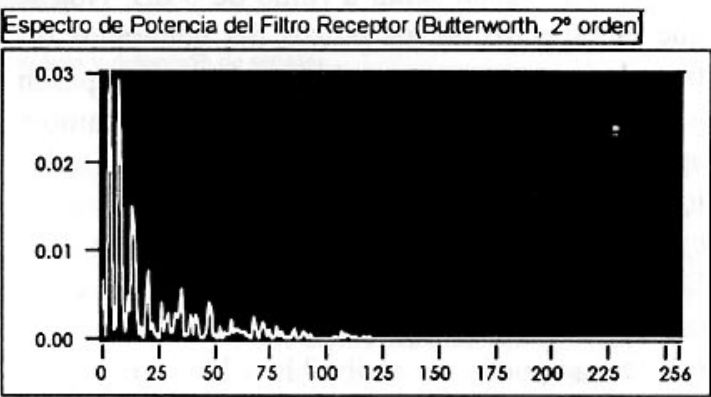

(d)

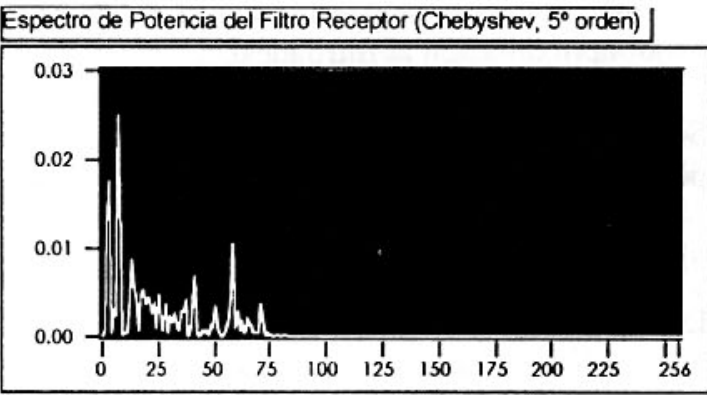

(f)

Figura No. 7: Espectros de potencia para el caso de la razón señal a ruido igual a $0 \mathrm{~dB}$, ancho de pulso de 20 UT y separación entre pulsos de 50 UT. (a) Pulsos del generador; (b) Ruido gaussiano; (c) Filtro transmisor de Butterworth, orden 2; (d) Filtro receptor de Butterworth de orden 2; (e) y (f) Filtros receptores de Chebyshev de orden 2 y 5 , respectivamente. 
Fourier (FFT) a una secuencia de datos de modo que:

$$
S_{X X}=\frac{1}{n^{2}}|F(X)|^{2}
$$

donde $S_{x x}$ es la secuencia de salida del espectro de potencia y $n$ representa el número de muestras en la secuencia de entrada $X$. En todos los casos analizados se tiene $n=512=2^{9}$, por lo que el cálculo del espectro de potencia se facilita. Hay que aclarar que la potencia total del $i$-ésimo armónico (excluyendo la componente DC que está en $f=0$ y el armónico de Nyquist en $\dot{f}=n / 2=256$ ) es igual al doble del valor graficado en los oscilogramas. La componente DC y el armónico de Nyquist no se multiplican por dos.

La figura No. 7 muestra varios casos de espectros de potencia, aunque todos tienen en común una razón señal a ruido de $0 \mathrm{~dB}$. Nótese que el espectro de los pulsos del generador (a) tiene bajos componentes de frecuencia que pasan a través del filtro transmisor (c) sin cambio apreciable. El espectro del ruido (b) tiene todos los componentes de frecuencia, como era de esperarse. Se puede ver que el filtro receptor de Butterworth de orden 2 (d) tiene menos componentes que el equivalente de Chebyshev (e), lo que puede ser atribuible a la interferencia entre símbolos. Nótese que el desempeño de Chebyshev mejora para un orden mayor del filtro (f), como era de esperarse pues existe una mejor aproximación al filtro ideal.

Se puede notar que ninguno de los espectros posee componente DC.

\section{CONCLUSIONES}

Del análisis de los resultados obtenidos utilizando el instrumento desarrollado en LabVIEW se desprende que las simulaciones entregan datos bastante confiables en su desempeño. Se logró hacer algunas comparaciones entre los filtros de Butterworth y
Chebyshev y se concluye que los primeros parecen tener mejor desempeño a bajos órdenes. Esto se puede deber a que los segundos presentan el fenómeno de interferencia entre símbolos.

La probabilidad de error parece seguir una función del tipo complementaria de error, con tendencia a aproximarse a 0.5 para valores muy bajos de la razón señal a ruido. Las probabilidades caen por debajo de $10^{-4}$ más rápidamente para filtros de Butterworth de orden 2 con razones superiores a los $5 \mathrm{~dB}$.

Los espectros de potencia muestran que ninguna señal contiene componentes DC ni de Nyquist, salvo por el ruido. De nuevo, se observó un mejor desempeño para filtros Butterworth de orden 2 que para su similar de Chebyshev.

\section{AGRADECIMIENTO}

El autor desea agradecer al Ing. E. Navas por su guía a lo largo de este trabajo.

\section{REFERENCIAS}

[1]. K. Sam Shanmugam, "Digital and Analog Communication Systems". (John Wiley \& Sons, New York) 1979.

[2]. M. Spiegel, "Manual de fórmulas y tablas matemáticas". (McGraw-Hill, México) 1985.

[3]. National Instruments, "LabVIEW 2 Analysis VI Library Reference Manual". (Austin, Texas) 1992.

Arturo Ramírez P., Investigador del Centro de Investigación en Ciencias e Ingeniería de Materiales (CICIMA) y estudiante de maestría en Sistemas Digitales de Ingeniería Eléctrica. 